\title{
Etre âgé à l'âge du numérique, défis et opportunités
}

Antoine Geissbuhler

A l'ère du numérique, les patients et les professionnels découvrent de nouveaux outils qui modifient les attitudes et comportements vis-à-vis des soins et de la santé. Ces outils de la cybersanté offrent de nouvelles possibilités d'autonomiser les patients et de sécuriser les transitions dans nos systèmes de santé complexes (Topol, 2014 ; Geissbuhler, 2012). Ils se déploient à plusieurs niveaux.

\section{Senioren im digitalen Zeitalter - Herausforderungen und Chancen}

Im digitalen Zeitalter entdecken Patienten und Angehörige der Pflegeberufe neue Instrumente, welche die Einstellungen und Verhaltensweisen gegenüber Pflege und Gesundheit verändern. Diese Instrumente der Cybergesundheit eröffnen neue Möglichkeiten, um Patienten zu mehr Selbstständigkeit zu verhelfen und die Übergänge in unseren komplexen Gesundheitssystemen zu sichern (Topol, 2014; Geissbuhler, 2012). Sie kommen auf mehreren Ebenen zum Einsatz.

DOl 10.1024/2297-5160/a000029

\section{Nouveaux outils au domicile}

Tout d'abord, au domicile du patient, avec l'irruption des technologies d'assistance visant à améliorer l'autonomie, la sécurité et la qualité de vie. Ces capteurs connectés, ces meubles et logements intelligents, ces nouveaux outils de communication qui font fi des distances, et même ces robots d'assistance suscitent des questionnements importants autour de la protection de la sphère privée, des liens et rôles familiaux, ainsi que de nouvelles manières de prodiguer des soins de santé, notamment à distance avec la télémédecine.

Ensuite, dans le réseau de soins, avec le développement de la cybersanté au niveau Suisse et des projets pionniers tels que le réseau genevois MonDossierMédical.ch. Il s'agit là de rassembler et d'outiller les différents intervenants,

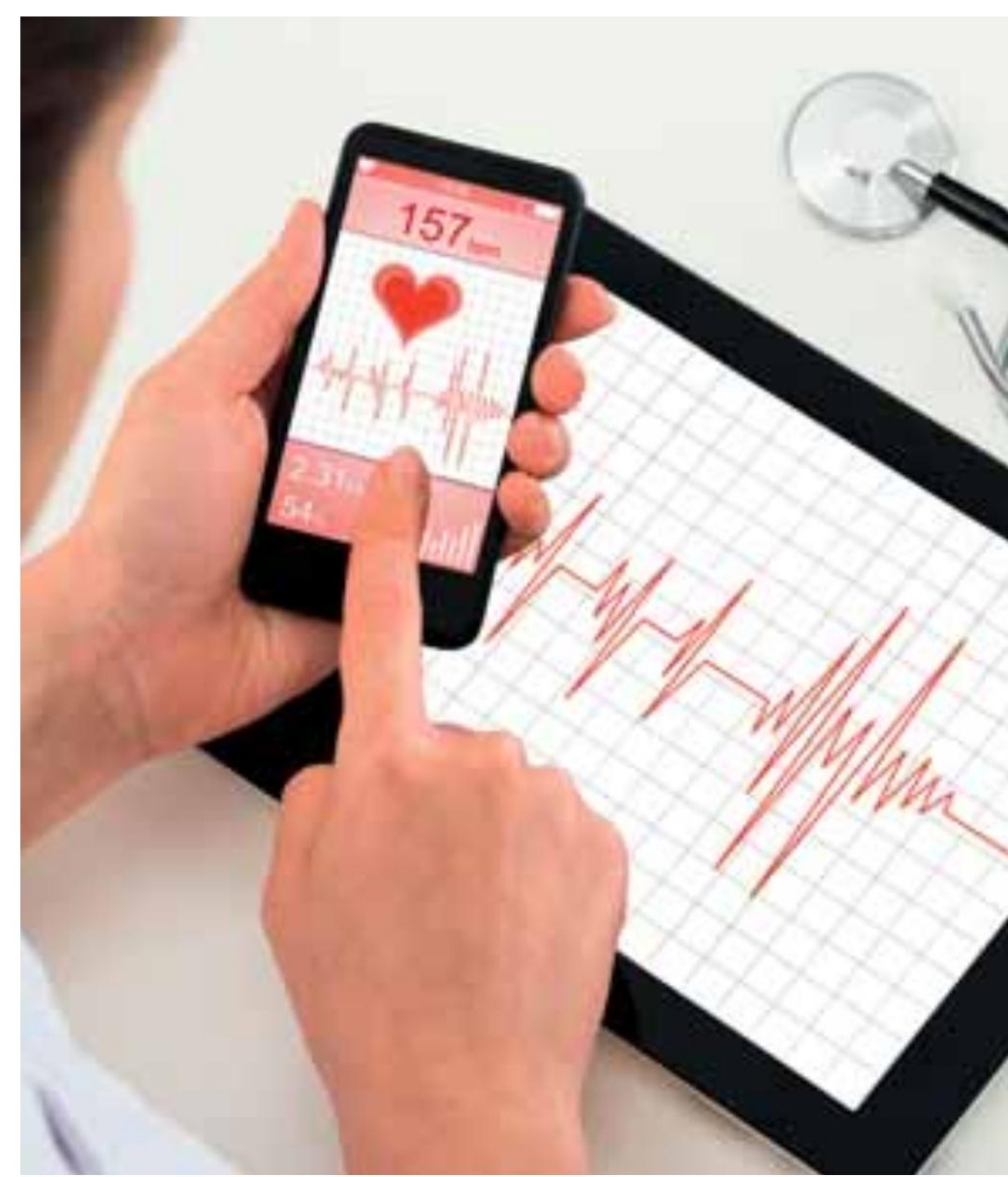

patients, proches, professionnels, pour l'organisation de la prise en charge multidisciplinaire des patients complexes et chroniques. De nouveau, au-delà des enjeux technologiques, il s'agit de réfléchir aux implications sociétales, à la responsabilité individuelle vis-à-vis de sa santé, à la participation accrue des proches aidants, ainsi qu'à la réorganisation des soins ( Geissbuhler, 2013).

Enfin, à l'hôpital, avec une réflexion nécessaire sur sa place dans un réseau de soins qui doit se centrer sur le patient, plutôt qu'être un lieu organisé autour des professionnels de la santé. Cela dans un contexte d'augmentation rapide des besoins en soins de santé d'une population vieillissante mais de ressources financières contraintes. Comment être à l'écoute des valeurs des patients et de leurs proches, et comment les intégrer véritablement dans l'évolution des soins hospitaliers en réseau ? Faut-il agran- 
dir les hôpitaux ou les « dématérialiser » pour les rendre efficaces aussi au domicile du patient ? Quels types de partenariats doivent être tissés pour favoriser l'innovation en phase avec les besoins des patients ? Comment les rôles professionnels vont-ils évoluer et comment va-t-on former les nouvelles professions nécessaires pour l'hôpital de demain ? L'élaboration du plan stratégique 2015-2020 des Hôpitaux Universitaires de Genève aborde quelques-unes de ces questions et envisage différentes actions pour y répondre ( Plan stratégique 2015-2020, HUG, 2012 ).

Si le système de santé ne peut se réduire à ces trois acteurs, et si d'autres développements, notamment autour de la médecine personnalisée sont appelés à y prendre une place grandissante, ces perspectives illustrent bien les enjeux actuels techniques, éthiques, humains et sociétaux, mais aussi les opportunités de l'intégration des outils du numérique pour la santé et les soins des personnes âgées, au moment où la stratégie nationale de cybersanté entre dans sa phase d'implémentation ( Stratégie Cybersanté Suisse, OFS 2007).

\section{Références}

Topol E. (2014) The Patient Will See You Now: The Future of Medicine is in Your Hands. Basic eds.

Geissbuhler A. (2012) eHealth: easing transitions in health care. Swiss Med Wkly., 142, w13599.

Geissbuhler A. (2013) Lessons learned implementing a regional health information exchange in Geneva as a pilot for the Swiss national eHealth strategy. Int J Med Inform, May 82(5), e118-24.

http://www.hug-ge.ch/document/plan-strategique-2015-2020 [visité le 13 septembre 2015].

http://www.bag.admin.ch/themen/gesundheitspolitik/10357/ 10359/index.html?lang=fr [visité le 13 septembre 2015].

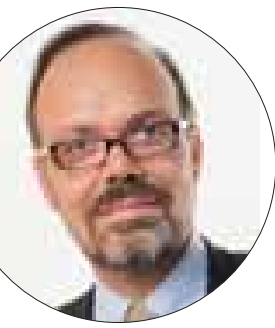

Prof. Dr. Antoine Geissbuhler est médecin-chef du service de cybersanté et télémédecine des Hôpitaux Universitaires de Genève et directeur du département de radiologie et informatique médicale de la Faculté de Médecine de l'Université de Genève.

antoine.geissbuhler@hcuge.ch

\section{Schon vergessen, was Sie gelesen haben?}

